\title{
O uso da cavitação hidrodinâmica aplicado ao tratamento de água
}

\author{
Use of the cavitation hydrodynamics applied to water treatment
}

\section{Eliezer Fares Abdala Neto', Marisete Dantas Aquino², Jefferson Pereira Ribeiro', Carla Bastos Vidal', Ronaldo Ferreira do Nascimento ${ }^{3}$, Franscico Wagner de Sousa ${ }^{4}$}

\begin{abstract}
RESUMO
A cavitação hidrodinâmica se apresenta como um processo oxidativo avançado (POA), permitindo integrar-se aos processos de tratamento de água para consumo humano. Esta pesquisa teve como objetivo investigar os efeitos de diferentes reatores de cavitação hidrodinâmica para tratar água contaminada com esgoto doméstico, avaliando a contaminação microbiológica e os parâmetros físico-químicos. Foram construídos três modelos de reatores com diferentes rotores (R1, R2 e R3). Os resultados obtidos nos reatores R1, R2 e R3 mostraram redução nas concentrações dos parâmetros químicos em todas as amostras, particularmente nitritos, nitratos, bicarbonatos e dureza total, ao passo que a contaminação microbiológica teve inativação completa dos microrganismos estudados (coliformes totais e Escherichia coli).
\end{abstract}

Palavras-chave: cavitação hidrodinâmica; processos oxidativos avançados (POA); tratamento de água.

\begin{abstract}
The hydrodynamic cavitation is presented as an advanced oxidation process (AOP), allowing to integrate the processes of water treatment for human consumption. This research aimed to investigate the effects of different hydrodynamic cavitation reactors for treating water contaminated with sewage, evaluating the microbiological contamination and physicochemical parameters. Three models were constructed with different rotors reactors (R1, R2 and R3). The results obtained in the reactors R1, R2 and R3 have shown a reduction in the concentrations of chemical parameters in all samples, particularly nitrites, nitrates, bicarbonates, and total hardness, while the microbiological contamination was completely inactivation (total coliforms and Escherichia coli).
\end{abstract}

Keywords: hydrodynamic cavitation; advanced oxidation processes (AOP); treatment of water.

\section{INTRODUÇÃO}

A cavitação foi primeiramente analisada e descrita por Reynolds em 1840 (CAUPIN \& HERBERT, 2006); apesar de ser amplamente estudada como um fenômeno danoso para máquinas hidráulicas, por outro lado pode ser inserida no rol das técnicas físicas para desinfecção de água, dispensando o uso de cloro e ou derivados clorados. Dessa forma, apresenta-se como alternativa promissora, pois permite integrar-se aos processos de tratamento de água para consumo humano (PASCHOALATO et al., 2008).
Dentre os vários processos de desinfecção utilizados para tratamento de água, os que utilizam cloro como agente desinfetante prevalecem. Entretanto, a reação do cloro com ácidos fúlvicos e húmicos presentes na água leva a formação dos trihalometanos (THM), reconhecidos como poderosos depressores da saúde humana e cancerígenos (FERREIRA FILHO \& SAKAGUTI, 2008; PASCHOALATO et al., 2008; SOUZA \& DANIEL, 2005).

O uso de técnicas híbridas para tratamento de água, sem o uso de cloro, com destaque para a combinação de radiação UV/ozonização,

'Doutorando em Engenharia Civil/Saneamento Ambiental pela Universidade Federal do Ceará (UFC) - Fortaleza (CE), Brasil.

2Doutora em Meio Ambiente e Recursos Hídricos pelo École des Hautes Études en Sciences Sociales - Paris, França. Professora do departamento de Engenharia Hidráulica e Ambiental da UFC - Fortaleza (CE), Brasil.

${ }^{3}$ Doutor em Química Analítica pela Universidade Federal de São Carlos (Ufscar) - São Carlos (SP), Brasil. Professor do Departamento de Engenharia Hidráulica e Ambiental da UFC - Fortaleza (CE), Brasil.

${ }^{4}$ Doutor em Engenharia Civil/Saneamento Ambiental pela UFC - Fortaleza (CE), Brasil. Professor do Departamento de Química do Instituto Federal de Educação, Ciência e Energia (IFCE) - Crateús (CE), Brasil.

Endereço para correspondência: Francisco W. de Sousa - Rua Lopes Vieira, s/n - 63700-000 - Crateús (CE), Brasil - E-mail: fr.wagner@ifce.edu.br

Recebido: 01/04/12 - Aceito: 27/09/13 - Reg. ABES: 314 
ultrassom/ozonização e UV/POA, vem se destacando nos últimos anos (JYOTI \& PANDIT, 2004; PASCHOALATO et al., 2008).

Em contrapartida, uma técnica alternativa de desinfecção que vem despontando no cenário mundial para tratamento de água é a cavitação. A cavitação é um processo oxidativo avançado (POA) bastante utilizado no tratamento de água e efluentes, caracterizada pela geração de cavidades, com posterior crescimento e colapso de bolhas que liberam grandes quantidades de energia (DELMÉE, 2003; CAUPIN \& HERBERT 2006; GOGATE, 2007; JYOTI \& PANDIT, 2004). Os principais efeitos dos fenômenos de cavitação são a geração de condições de temperatura e pressão muito elevadas com liberação de radicais livres altamente reativos e de geração de microturbulências, aumentando assim as taxas de fenômenos de transporte (DELMÉE, 2003; JYOTI \& PANDIT, 2004).

O fenômeno de cavitação pode ocorrer de duas formas: uma devido à passagem de ondas sonoras de alta frequência, chamado cavitação acústica (ultrassonificação), e outra devido a variações de pressão no líquido, que flui graças à mudança na geometria no sistema de fluxo, chamado cavitação hidrodinâmica (DELMÉE, 2003; JYOTI \& PANDIT, 2004).

Os reatores de cavitação hidrodinâmica têm amplo uso nos processos de desinfecção de água, (MASON, 1992). A literatura relata diversos trabalhos que usam a cavitação hidrodinâmica para tratamento de água (ARROJO \& BENITO, 2008; JYOTI \& PANDIT, 2001).

O presente trabalho teve como objetivo investigar os efeitos de diferentes geometrias de reatores de cavitação hidrodinâmica para desinfecção de água contaminada com esgoto doméstico avaliando a concentração de indicadores de contaminação microbiológica (coliformes totais e Escherichia coli) e parâmetros físico-químicos.

\section{METODOLOGIA}

\section{Local dos experimentos}

A água utilizada nos experimentos foi proveniente de um poço localizado na estação climatológica da Universidade Federal do Ceará (UFC), com profundidade de $49 \mathrm{~m}$, nível estático de $23 \mathrm{~m}$, nível dinâmico de $43 \mathrm{~m}$ e vazão de $4.200 \mathrm{~L} \cdot \mathrm{h}^{-1}$. Esse poço foi escolhido por se localizar nas proximidades do local das instalações experimentais, nas dependências do Núcleo de Tecnologia Industrial do Ceará (NUTEC), e também por atender aos padrões de potabilidade vigente, Portaria $\mathrm{n}^{\circ} 518$ do Ministério da Saúde do Brasil (BRASIL, 2005).

\section{Preparação das amostras}

Um tanque com capacidade de $1.500 \mathrm{~L}$ foi utilizado para reservar água bruta coletada em cada um dos dias de experimento. A esse volume foram realizadas inoculações de esgoto bruto doméstico, coletado em um poço de visita da Companhia de Água e Esgoto do Estado do Ceará (CAGECE), localizada no interior do câmpus da UFC.

Foram coletados $20 \mathrm{~L}$ de esgoto bruto divididos em 4 coletas ao longo do dia, nos seguintes horários: 08, 11, 14 e finalizando às $17 \mathrm{~h}$, com o acondicionamento sob refrigeração. Dessa forma, buscaram-se os horários de pico com o objetivo de obter maior concentração microbiológica nas amostras. No dia seguinte, as amostras de esgoto foram homogeneizadas e separou-se uma alíquota de 4,8 L para ser adicionada a água bruta. Na Tabela 1 estão apresentados os resultados médios das características da água bruta, com e sem inoculação de esgoto bruto doméstico.

\section{A concepção do reator de cavitação hidrodinâmica}

A Figura 1 apresenta o esboço das peças que compõem os reatores construídos e os instrumentos de monitoramento: pressão, temperatura e rotação dos rotores devidamente instalados. Foram construídos três modelos de reatores (R1, R2 e R3), com diferentes números de orifícios usinados na face superior do rotor, única peça móvel do equipamento responsável pelo efeito de cavitação hidrodinâmica aplicada ao líquido, portanto as demais não sofreram alterações dimensionais.

Tabela 1 - Valores médios para os parâmetros físico-químicos e microbiológicos da água bruta, com e sem a inoculação de esgoto bruto.

\begin{tabular}{|c|c|c|}
\hline Características & Água bruta & $\begin{array}{c}\text { Água bruta } \\
\text { com inoculação }\end{array}$ \\
\hline $\mathrm{pH}$ a $25^{\circ} \mathrm{C}$ & 7,91 & 8,07 \\
\hline Condutividade (micromhos.cm') & 1012,00 & 964,00 \\
\hline Alcalinidade parcial $\left(\mathrm{mgCaCO}_{3} \mathrm{~L}^{-1}\right)$ & 2,00 & 10,90 \\
\hline Alcalinidade total $\left(\mathrm{mgCaCO}_{3} \mathrm{~L}^{\mathrm{L}}{ }^{-1}\right)$ & 254,40 & 248,50 \\
\hline Dureza total $\left(\mathrm{mgCaCO}_{3} \cdot \mathrm{L}^{-1}\right)$ & 156,00 & 145,10 \\
\hline Matéria orgânica $\left(\mathrm{mgO}_{2} \cdot \mathrm{L}^{-1}\right)$ & $\mathrm{Nd}$ & 0,60 \\
\hline Nitritos (mgN-NO $\left.{ }_{2} \mathrm{~L}^{-1}\right)$ & 0,01 & 0,30 \\
\hline Sólidos dissolvidos (mg.:-1') & 758,00 & 721,00 \\
\hline Sólidos totais (mg.L') & 842,00 & 834,00 \\
\hline Cálcio $\left(\mathrm{mgCa}^{2+} \cdot \mathrm{L}^{-1}\right)$ & 11,40 & 8,40 \\
\hline Magnésio $\left(\mathrm{mgMg}^{2+} \cdot \mathrm{L}^{-1}\right)$ & 31,00 & 30,20 \\
\hline Sódio $\left(\mathrm{mgNa}^{+} \mathrm{L}^{-1}\right)$ & 160,00 & 158,00 \\
\hline Potássio $\left(\mathrm{mgK}^{+} . \mathrm{L}^{-1}\right)$ & 30,70 & 28,00 \\
\hline Ferro total (mgFe. $\left.L^{-1}\right)$ & 0,20 & 0,90 \\
\hline Cloretos (mgCl:.'ㄹ) & 157,40 & 157,90 \\
\hline Carbonatos $\left(\mathrm{mgCO}_{3}{ }^{2 \cdot} \cdot \mathrm{L}^{-1}\right)$ & 2,40 & 13,10 \\
\hline Bicarbonatos $\left(\mathrm{mgHCO}_{3} \mathrm{LL}^{-1}\right)$ & 305,60 & 276,60 \\
\hline Hidróxidos (mgOH:L'1) & $\mathrm{Nd}$ & $\mathrm{Nd}$ \\
\hline Sulfatos $\left(\mathrm{mgSO}_{4}^{\left.2-\mathrm{L}^{-1}\right)}\right.$ & 54,20 & 43,90 \\
\hline 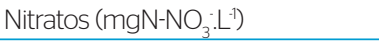 & 3,10 & 9,60 \\
\hline Coliformes totais (NPM.100mL'ㄱ) & $2,89 \times 10$ & $2,85 \times 10^{6}$ \\
\hline Escherichia coli (NPM.10OmL-1') & $1,20 \times 10$ & $6,27 \times 10^{5}$ \\
\hline
\end{tabular}

Nd: não detectado. 
O reator foi construído com eixo em aço inox 304, medindo $50,8 \mathrm{~mm}$ de diâmetro e comprimento de $400,0 \mathrm{~mm}$. O rotor foi usinado em alumínio com $508 \mathrm{~mm}$ de diâmetro e $80 \mathrm{~mm}$ de espessura, e os orifícios mediam $18 \mathrm{~mm}$ de diâmetro e tinham profundidade de $150 \mathrm{~mm}$. O diferencial empregado se deu nas quantidades de orifícios usinados nos rotores: para o rotor 1 (R1), foram usinados 14 orifícios; para o rotor 2 (R2), 20 orifícios; e para o rotor 3 (R3), 26 orifícios. Em todos os rotores construídos os orifícios foram distribuídos uniformemente em sua face superior. A diferença entre o diâmetro interno do estator e o diâmetro externo do rotor ficou em 1,5 mm.

Para a seleção da velocidade de rotação, optou-se por seguir os resultados obtidos por Jyoti e Pandit (2001), os quais realizaram experimentos conduzindo um reator de cavitação. Os resultados mostraram que, operando em baixas rotações, ocorreu baixa taxa de desinfecção, à medida que a velocidade aumentava, o índice de desinfecção também aumentava. Esse efeito foi observado entre 4.000 e $8.000 \mathrm{rpm}$. O efeito máximo observado foi $8.000 \mathrm{rpm}$, ao passo que, para velocidades acima de 8.000 até $12.000 \mathrm{rpm}$, ocorreu queda na taxa de desinfecção. Tendo como base esses parâmetros, o reator empregado nesta pesquisa operou com velocidade rotacional de $8.200 \mathrm{rpm}$, acionado por um motor elétrico (de marca WEG) de 20 KW, 6 polos, tensão nominal de $380 \mathrm{~V}-120 \mathrm{~Hz}$, acoplado a um inversor de frequência WEG-CFW 11. A pressão foi monitorada por um manômetro (marca Famabras - Série FSG), a temperatura por termômetro digital (Minipa), e para a velocidade rotacional foi usado um contador de giro (marca Racetronix).

\section{Configuração do experimento}

O aparato experimental foi montado conforme a Figura 2, com dois reservatórios (tanque 1 e 2), bomba hidráulica, registros hidráulicos, reator de cavitação e motor elétrico.

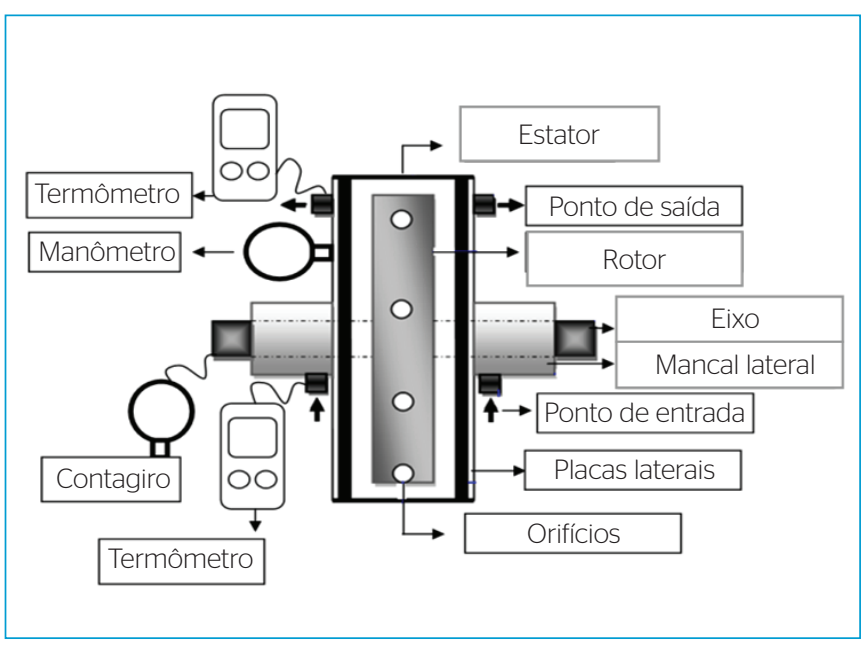

Figura 1 - Conjunto de componentes do reator de cavitação utilizado no estudo.
O tanque 1, com capacidade de 1.500 L (diâmetro de $1.830 \mathrm{~mm}$ e altura de $990 \mathrm{~mm}$ ), serviu ao mesmo tempo como reservatório de água bruta inoculada e para resfriamento da água tratada que era armazenada no tanque 2. Uma bomba hidráulica (Danfoss) operou com 0,25 kWh em $220 \mathrm{~V}$, com sucção de água direta do tanque 1. No recalque, por uma tubulação de cloreto de polivinila (PVC) com 3/4 de polegadas de diâmetro, havia dois ramais com registros hidráulicos, sendo um para alimentar o reator de cavitação e outro para retornar com o excedente ao tanque 1 , conforme seleção da vazão a ser experimentada, auxiliada por um rotâmetro (Tecnofluid) instalado na jusante do registro.

Após a água ser submetida ao reator de cavitação, seguia por uma tubulação de cobre de 1/4 de polegada de diâmetro e $6 \mathrm{~m}$ de comprimento, em espiral, posicionada submersa no tanque de água bruta no tanque 1, para condensar até sua chegada ao tanque 2 (capacidade de $200 \mathrm{~L}$ ), ao passo que a água bruta era preaquecida, auxiliando o processo.

Os ensaios com os reatores R1, R2 e R3 foram realizados em modo contínuo, divididos em duas etapas para cada modelo de reator. $\mathrm{Na} 1^{\text {a }}$ etapa, foram feitos 10 experimentos com os reatores operando com vazões de 40, 60, 80 e 100 L.h ${ }^{-1}$. Esses testes objetivaram avaliar a vazão que promove a ebulição da água de estudo em menor tempo de operação, visto que a otimização da velocidade de ebulição da água influi diretamente na eficiência de desinfecção, bem como na redução de consumo de energia do sistema. Para isso, foi realizado o ajuste da vazão de alimentação via rotâmetro e, em seguida, foi iniciada a operação do reator em modo contínuo por $39 \mathrm{~min}$. Esse tempo de operação se justifica devido aos resultados preliminares (em $35 \mathrm{~min}$ ) terem indicado uma constância na temperatura independente das vazões estudadas. Portanto, o intervalo de tempo de 39 min estipulado contempla a estabilização do processo.

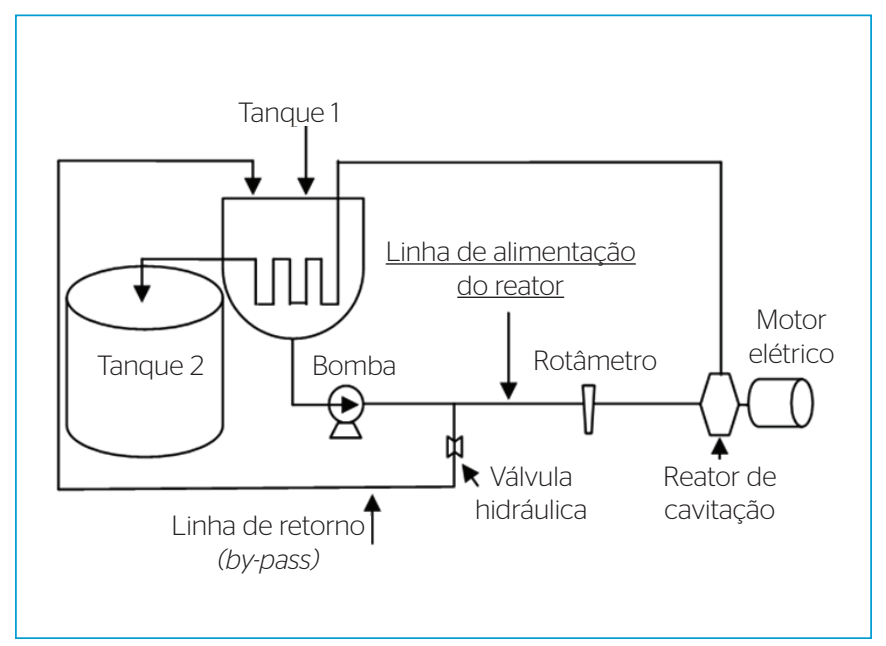

Figura 2 - Montagem do experimento com o reator de cavitação hidrodinâmica. 
O monitoramento da temperatura foi realizado por termopares instalados no ponto de entrada e saída da água do reator, medidos em intervalos de 3 min para melhor aferir o comportamento do sistema e as alterações de estado do fluido (perfazendo 13 coletas para cada experimento). No total foram analisadas 130 amostras referentes a $1^{\text {a }}$ etapa de experimentos para cada tipo de reator (R1, R2 e R3).

$\mathrm{Na} 2^{\text {a }}$ etapa dos experimentos, avaliou-se a desinfecção obtida para os indicadores de contaminação microbiológica (coliformes totais e Escherichia coli), com os reatores R1, R2 e R3 operando nas suas vazões de maior rendimento térmico (otimizadas). Realizaram-se 10 experimentos para cada um dos reatores, com amostras coletadas no início de cada um deles para água bruta e água bruta inoculada, e para água tratada a coleta foi realizada após 25 min de operação dos reatores. Amostras destinadas às análises físico-químicas foram coletadas em frascos de plásticos com capacidade de $1.500 \mathrm{~mL}$, e as amostras para os exames microbiológicos, em frascos esterilizados com capacidade de $100 \mathrm{~mL}$. Após as coletas, as amostras foram identificadas, refrigeradas em caixa de isopor e conduzidas para os laboratórios de química e microbiologia do Núcleo de Tecnologia Industrial do Ceará (NUTEC) e Laboratório de Saneamento Ambiental da Universidade Federal do Ceará (LABOSAN).

Todas as amostras utilizadas no presente trabalho não sofreram nenhum tratamento preliminar para realização deste estudo.

$\mathrm{Na}$ Tabela 2 estão apresentados os parâmetros e exames analisados com seus respectivos métodos analíticos empregados e referências.

\section{RESULTADOS E DISCUSSÃO}

\section{Otimização da vazão para cada tipo de reator experimentado}

Os resultados médios de temperatura obtidos com o uso dos diferentes reatores de cavitação nas vazões de 40, 60, 80 e 100 L.h h $^{-1}$ estão mostrados na Figura 3. Observou-se que o reator R1, nas condições em que foi dimensionado, conforme apresentado na Figura 3a, atingiu o pico máximo de temperatura de água tratada em $60^{\circ} \mathrm{C}$, estabilizando-se 30 min após o inicio da operação na vazão de 80 L.h.' . Ao passo que, no reator R2, a estabilidade foi atingida após $33 \mathrm{~min}$ de início de operação com vazão de $60 \mathrm{~L} \cdot \mathrm{h}^{-1}$, atingindo a máxima temperatura em $78^{\circ} \mathrm{C}$.

Para o reator R3, os resultados ressaltam que, para uma vazão de 40 L.h h $^{-1}$ o sistema entra em ebulição a 33 min de operação, ao passo que, na vazão de $60 \mathrm{~L} \cdot \mathrm{h}^{-1}$, a temperatura de $100^{\circ} \mathrm{C}$ foi estabilizada com 21 min do início da operação do sistema, conferindo-lhe a melhor condição de operação. Em contrapartida, para uma vazão de 80 L.h ${ }^{-1}$ a ebulição foi atingida em 30 min de operação, enquanto na vazão de 100 L.h ${ }^{-1}$ a produção de vapor não é alcançada de modo contínuo. A ausência de estabilização da temperatura de ebulição para a vazão de 100 L.h ${ }^{-1}$, pode ser devida à taxa de renovação de água dentro do reator, a qual pode impossibilitar a absorção de calor da parcela de líquido a ponto de mudar seu estado.

Segundo Suslick et al. (1999) e Gogate et al. (2006), em um ambiente de ondas cavitantes, com colapso das bolhas, podem ser produzidos pontos quentes com temperaturas efetivas e pressões bastantes elevadas,

Tabela 2 - Parâmetros e exames analisados com seus respectivos métodos analíticos empregados e referências.

\begin{tabular}{|c|c|c|}
\hline Parâmetros & Métodos analíticos & Referência \\
\hline Alcalinidade total $\left(\mathrm{mgCaCO}_{3} \mathrm{~L}^{-1}\right)$ & 2320 B Titration Method & APHA, 1998 \\
\hline Dureza total $\left(\mathrm{mgCaCO}_{3} \mathrm{~L}^{-1}\right)$ & 2340 C EDTA Tritimetric Method & APHA, 1998 \\
\hline Nitritos (mgN-NO$\left.{ }_{2}^{2} \cdot L^{-1}\right)$ & 4500 B Colorimetric Method & APHA, 1998 \\
\hline Nitratos(mgN-NO$\left.{ }_{3} \cdot \mathrm{L}^{-1}\right)$ & Método do Silicato de Sódio & Rodier, 1990 \\
\hline Cálcio(mgCa $\left.{ }^{2+} \cdot \mathrm{L}^{-1}\right)$ & 3500 Ca EDTA Titrimetric Method & APHA, 1998 \\
\hline Magnésio $\left(\mathrm{mgMg}^{2+} \cdot \mathrm{L}^{-1}\right)$ & 3500 Mg B Calculation Method & APHA, 1998 \\
\hline Sódio $\left(m g N a^{+} L^{-1}\right)$ & $3500 \mathrm{Na}$ B Flame emission photometric & APHA, 1998 \\
\hline Potássio $\left(\mathrm{mgK}^{+} \mathrm{L}^{-1}\right)$ & 3500 K B Flame photometric Method & APHA, 1998 \\
\hline Ferro total (mgFe. $\left.L^{-1}\right)$ & 3500 B Phenanthroline Method & APHA, 1998 \\
\hline Cloretos (mgCl: L-1) $^{-1}$ & $4500 \mathrm{Cl}$ B Argentometric Method & APHA, 1998 \\
\hline Bicarbonatos $\left(\mathrm{mgHCO}_{3}^{\prime} / \mathrm{L}\right)$ & 2320 B Titration Method & APHA, 1998 \\
\hline Sulfatos $\left(\mathrm{mg} \mathrm{SO}_{4}{ }^{2 \cdot L^{-1}}\right)$ & 4500 SO4- E Turbidimetric Method & APHA, 1998 \\
\hline Sólidos Totais (mg.Lำ) & 2540 Solids B Total solids dried at 103-1050 C & APHA, 1998 \\
\hline Condutividade (micromhos.cm') & 2510 B Laboratory Method & APHA, 1998 \\
\hline Oxigênio consumido (mg..'1) & Método perganométrico & Rodier, 1990 \\
\hline Coliformes totais (NPM.100mL'ㄱ) & 9221D & APHA, 1998 \\
\hline Escherichia coli(NPM.100mL'1) & $9221 \mathrm{E}$ & APHA, 1998 \\
\hline
\end{tabular}




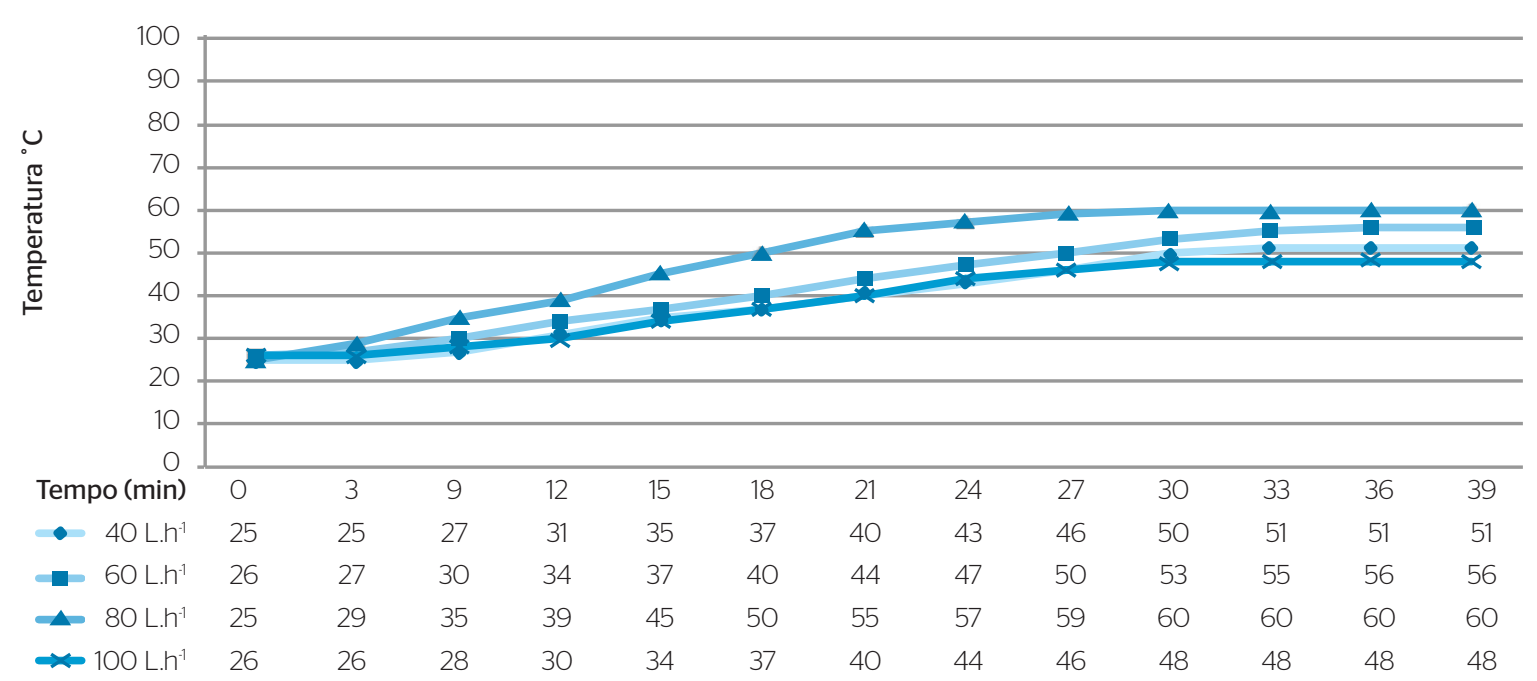

a) Valores médios de temperatura da água no reator R1 para as diferentes vazões experimentadas.

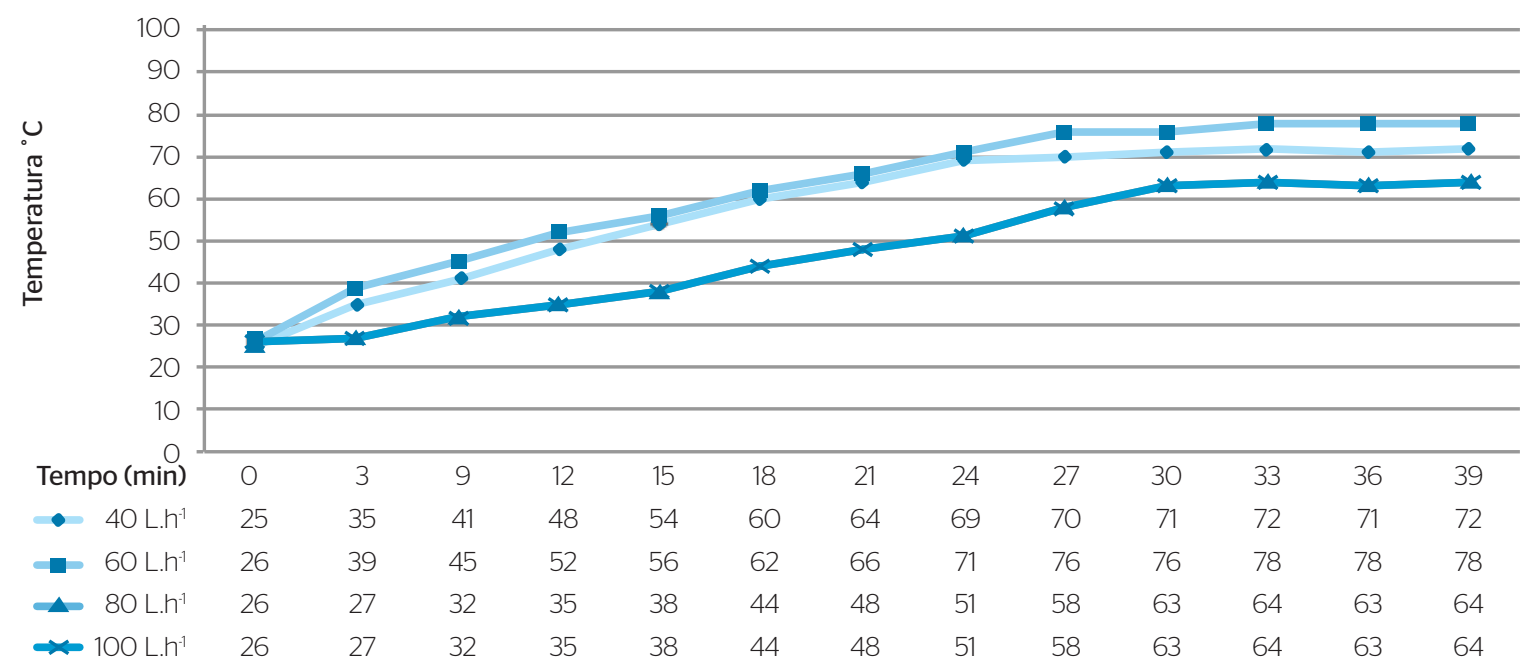

b) Valores médios de temperatura da água no reator R2 para as diferentes vazões experimentadas.

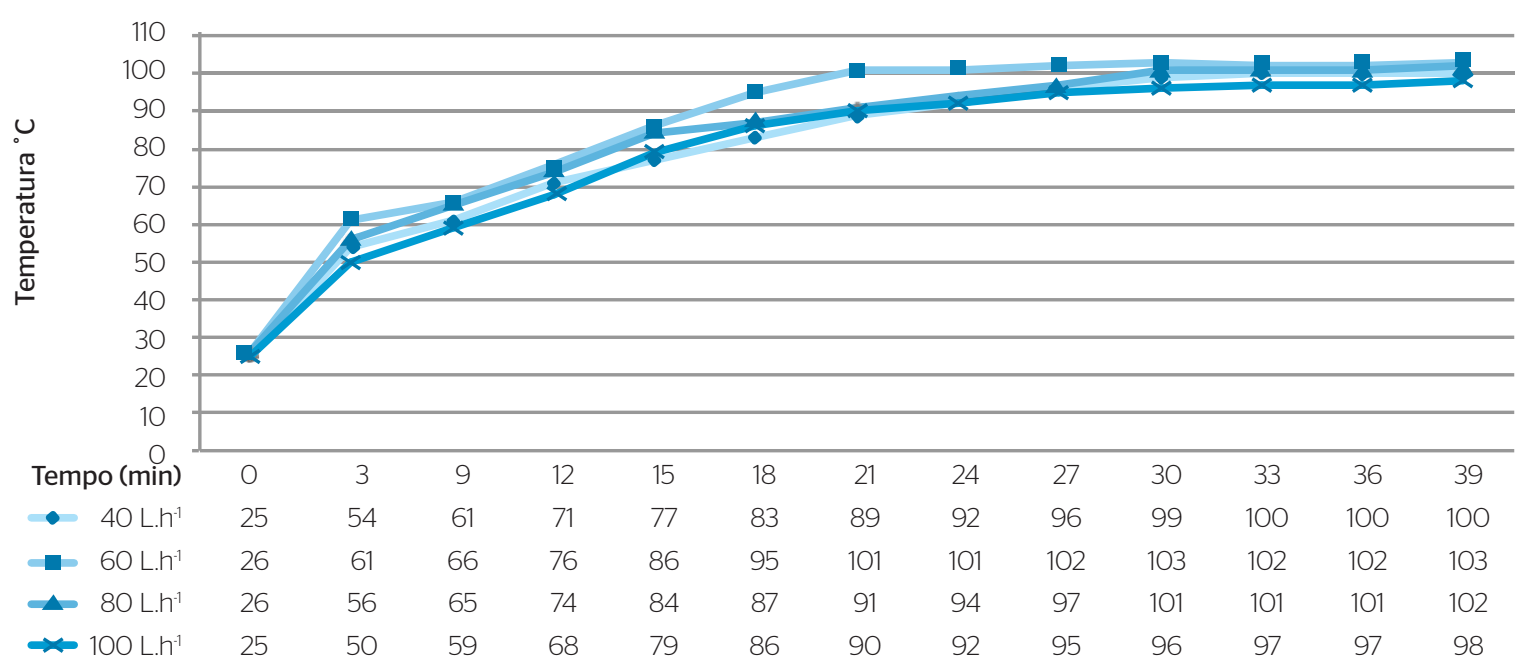

c) Valores médios de temperatura da água no reator R3 para as diferentes vazões experimentadas.

Figura 3 - Resultados médios de temperatura no ponto de saída de água tratada com R1, R2 e R3. 
bem como taxas de aquecimento e resfriamento extremamente rápidas. O reator de cavitação R3, operando com vazão de $60 \mathrm{~L} \cdot \mathrm{h}^{-1}$, apresentou o maior número de orifícios usinados no rotor e, possivelmente, potencializa maior formação, crescimento e subsequente colapso de microbolhas. Além disso, operando nas condições de vazão de 60 L.h ${ }^{-1}$, apresentou também melhor correspondência entre os colapsos das bolhas e a simultânea transmissão da energia térmica à massa líquida, bem como a taxa de renovação da água que alimenta o reator.

Os resultados obtidos com o monitoramento da pressão de descarga para o reator R3 são mostrados na Figura 4. Observa-se que nas vazões de 40, 80 e 100 L.h ${ }^{-1}$ houve pequenas variações nos valores de pressão, em comparação com os resultados com a vazão de 60 L.h ${ }^{-1}$, a qual atingiu o valor máximo em menor tempo.

Arrojo e Benito (2008) relataram que o acréscimo da pressão acelera o colapso das bolhas promovendo a difusão dos radicais hidroxilas $(\mathrm{OH})$ na fase líquida. Jyoti e Pandit (2004) relatam que o aumento da pressão torna o processo de desinfecção dos micro-organismos mais efetivo, bem como o rápido aumento da temperatura.

\section{Avaliação da qualidade físico-química e microbiológica da água bruta e tratada}

Para os reatores R1 e R2, observaram-se, conforme resultados apresentados na Tabela 1, alterações mínimas nos parâmetros físico-químicos estudados, indicando alterações não significativas nas características da água tratada.

Para o reator R3, operando a vazão de 60 L.h.' ${ }^{-1}$, os resultados referentes à determinação dos parâmetros físico-químicos indicam forte influência da água submetida aos fenômenos da cavitação hidrodinâmica, o que pode ser constatado nas alterações observadas nas características físico-químicas da água tratada. Em relação ao pH houve uma pequena diminuição, provavelmente pela oxidação de espécies orgânicas presentes na água, provocando consumo da alcalinidade durante o processo. Segundo Teixeira e Jardim (2004), o colapso das

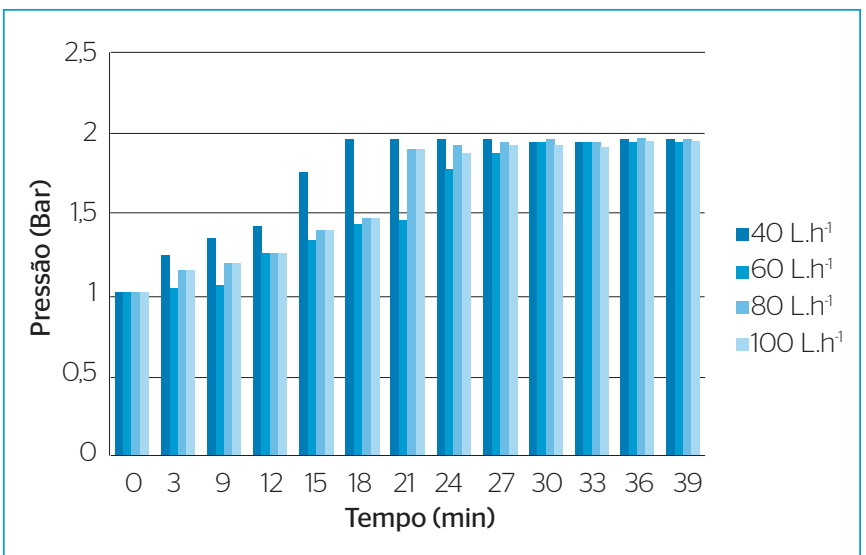

Figura 4 - Monitoramento da pressão medida no ambiente interno do reator de cavitação R3, operando nas vazões de 40, 60, 80 e 100 L.h'1. bolhas provoca a formação de átomos reativos de hidrogênio, os quais são responsáveis por promover reações de oxidação de compostos orgânicos. A redução da matéria orgânica pode ter sido provocada pelos radicais livres que proporcionam a oxidação dos compostos orgânicos. Esses átomos reativos podem ter provocado reduções consideráveis nos níveis de alcalinidade parcial, carbonatos, bicarbonatos e nitratos, conforme observado na Tabela 3. Também pode ser observado que o $\mathrm{pH}$ das amostras situaram-se entre 7 e 8 , indicando que a alcalinidade é à base de bicarbonato.

Teores de bicarbonatos, responsáveis pela dureza, foram reduzidos durante o processo devido à precipitação destes, o que pode ser verificado pelos baixos valores na água tratada. Devido à ausência de valores de referência da Portaria ${ }^{\circ} 518$ do Ministério da Saúde (BRASIL, 2005) para esse parâmetro, os valores finais da água tratada demonstram que o processo de cavitação hidrodinâmica mostra grande potencialidade na redução de bicarbonatos, possivelmente atribuído ao aumento da temperatura da água e diminuição da solubilidade.

A Tabela 4 apresenta os resultados obtidos para os indicadores de contaminação microbiológica (coliformes totais e Escherichia coli), com os reatores operando em suas vazões de maior rendimento térmico (otimizadas).

Para as condições de dimensionamento aplicadas nos reatores R1 e R2, operando com vazão otimizada de 80 e 60 L.h ${ }^{-1}$, respectivamente, observou-se que, embora tenha ocorrido decaimento na concentração de coliformes totais e Escherichia coli para as amostras de água tratada analisadas, os resultados obtidos apresentaram-se sempre acima do que preconiza a Portaria n 518 do Ministério da Saúde do Brasil (BRASIL, 2005), portanto atestando a baixa eficiência dos modelos de reatores $\mathrm{R} 1$ e $\mathrm{R} 2$.

Para o reator R3, operando com vazão de 60 L.h ${ }^{-1}$, observou-se que os valores obtidos para a água tratada se enquadraram dentro da faixa estabelecida pela legislação vigente no Brasil (BRASIL, 2005), evidenciando que o tratamento é eficaz para eliminação dos patógenos estudados. Essa eficiência na desinfecção pode ser atribuída à ruptura das células dos micro-organismos, submetidas ao intenso efeito da cavitação no líquido (JYOTI \& PANDIT, 2004), o qual gera temperaturas e pressões elevadas no ambiente interno do líquido (GOGATE et al., 2001). Outro fator que pode ter contribuído para esse resultado é a temperatura na faixa de ebulição $\left(100^{\circ} \mathrm{C}\right)$, que dessa forma impõem aos micro-organismos uma maior condição de desnaturação de suas proteínas provocando sua morte.

Jyoti e Pandit (2003) alcançaram um percentual de desinfecção de $70 \%$ para coliformes totais e $57 \%$ para coliformes fecais utilizando cavitação hidrodinâmica com placa com múltiplos orifícios em uma pressão de 1,72 bar. Doulah e Hammond (1975) sugeriram que um mecanismo de movimentos circulares em um líquido induz que as células entrem em ressonância. Quando esses movimentos circulares 
Tabela 3 - Características físico-químicas da água bruta, água bruta inoculada e água tratada para os diferentes reatores de cavitação hidrodinâmica operando com suas vazões otimizadas.

\begin{tabular}{|c|c|c|c|c|c|c|}
\hline \multirow[b]{2}{*}{ Características } & \multirow[b]{2}{*}{ Água bruta } & \multirow{2}{*}{$\begin{array}{l}\text { Água bruta } \\
\text { inoculada }\end{array}$} & \multicolumn{3}{|c|}{ Água tratada } & \multirow{2}{*}{$\begin{array}{c}\text { Valores de referência } \\
\text { Portaria N } 518 \text { (BRASIL, 2005) }\end{array}$} \\
\hline & & & $\begin{array}{c}\text { Reator R1 } \\
40 \text { L.h.' }\end{array}$ & $\begin{array}{c}\text { Reator R2 } \\
40 \mathrm{~L} . \mathrm{h}^{-1}\end{array}$ & $\begin{array}{c}\text { Reator R3 } \\
60 \text { L.h. }\end{array}$ & \\
\hline $\mathrm{pH}$ a $25^{\circ} \mathrm{C}$ & 7,91 & 8,07 & 8,0 & 7,55 & 7,15 & 6,0 a 9,5 \\
\hline Condutividade (micromhos.cm') & 1012,00 & 964,00 & 964,0 & 988,00 & 860,00 & - \\
\hline Alcalinidade parcial $\left(\mathrm{mgCaCO}_{3} \mathrm{~L}^{-1}\right)$ & 2,00 & 10,90 & 7,8 & 9,00 & $\mathrm{Nd}$ & - \\
\hline Alcalinidade total $\left(\mathrm{mgCaCO}_{3} \mathrm{~L}^{\mathrm{L}}{ }^{-1}\right)$ & 254,40 & 248,50 & 239,9 & 213,70 & 163,40 & - \\
\hline Oxigênio consumido $\left(\mathrm{mgO}_{2} \mathrm{~L}^{\mathrm{L}} \mathrm{L}^{-1}\right)$ & $\mathrm{Nd}$ & 0,60 & $\mathrm{Nd}$ & $\mathrm{Nd}$ & $\mathrm{Nd}$ & - \\
\hline Nitritos $\left(\mathrm{mgN}-\mathrm{NO}_{2} \cdot \mathrm{L}^{-1}\right)$ & 0,01 & 0,30 & 0,2 & 0,30 & 0,04 & $1,0 \mathrm{mg} \cdot \mathrm{L}^{-1}$ \\
\hline Sólidos dissolvidos (mg. $\mathrm{L}^{-1}$ ) & 758,00 & 721,00 & 750,4 & 742,00 & 602,00 & 1000,0 mg.L.1 \\
\hline Sólidos totais (mg.L') & 842,00 & 834,00 & 822,1 & 847,50 & 796,00 & - \\
\hline Cálcio $\left(\mathrm{mgCa}^{2+} \cdot \mathrm{L}^{-1}\right)$ & 11,40 & 8,40 & 9,0 & 9,40 & 8,40 & - \\
\hline Ferro total (mgFe. $\left.L^{-1}\right)$ & 0,20 & 0,90 & 0,9 & 0,80 & 0,20 & $0,3 \mathrm{mg}^{\mathrm{L}} \mathrm{L}^{-1}$ \\
\hline Cloretos (mgCl:L') & 157,40 & 157,90 & 155,8 & 158,00 & 153,20 & 250,0 mg. $^{-1}$ \\
\hline Carbonatos $\left(\mathrm{mgCO}_{3}{ }^{2} \cdot \mathrm{L}^{-1}\right)$ & 2,40 & 13,10 & $\mathrm{Nd}$ & $\mathrm{Nd}$ & $\mathrm{Nd}$ & - \\
\hline Bicarbonatos $\left(\mathrm{mgHCO}_{3} \mathrm{~L}^{-1}\right)$ & 305,60 & 276,60 & 300,2 & 298,50 & 199,30 & - \\
\hline Hidróxidos (mgOH:L'1') & $\mathrm{Nd}$ & $\mathrm{Nd}$ & $\mathrm{Nd}$ & $\mathrm{Nd}$ & $\mathrm{Nd}$ & - \\
\hline Sulfatos $\left(\mathrm{mgSO}_{4}^{2 \cdot} \cdot \mathrm{L}^{-1}\right)$ & 54,20 & 43,90 & 50,5 & 56,20 & 46,50 & 250,0 mg. $\mathrm{L}^{-1}$ \\
\hline Nitratos $\left(\mathrm{mgN}-\mathrm{NO}_{3} \cdot \mathrm{L}^{-1}\right)$ & 3,10 & 9,60 & 9,4 & 8,70 & 1,10 & $10,0 \mathrm{mg} \cdot \mathrm{L}^{-1}$ \\
\hline
\end{tabular}

$(-)$ não regulamentado; Nd: não detectado.

Tabela 4 - Resultados dos exames microbiológicos para amostras de água bruta inoculada e água tratada durante os experimentos com os reatores R1, R2 e R3 operando com suas vazões otimizadas.

\begin{tabular}{|c|c|c|c|c|c|}
\hline \multirow{3}{*}{ Testes } & \multirow{3}{*}{ Parâmetros } & \multirow{3}{*}{ Água bruta inoculada } & \multicolumn{3}{|c|}{ Agua tratada } \\
\hline & & & R1 & $\mathrm{R} 2$ & R3 \\
\hline & & & 80 L.h.' & 60 L.h.' & 60 L.h h $^{-1}$ \\
\hline Teste 1 & Escherichia coli (NPM/100 mL-1) & $36,4 \times 10^{4}$ & $8,7 \times 10^{4}$ & $1,7 \times 10^{2}$ & $\mathrm{Nd}$ \\
\hline \multirow{2}{*}{ Teste 2} & Coliformes totais (NPM.100 mL'1) & $2,9 \times 10^{6}$ & $9,6 \times 10^{4}$ & $5,2 \times 10^{5}$ & $\mathrm{Nd}$ \\
\hline & Escherichia coli (NPM/100 mL-1') & $1,4 \times 10^{6}$ & $5,4 \times 10^{5}$ & $1,7 \times 10^{4}$ & $\mathrm{Nd}$ \\
\hline Teste 3 & Escherichia coli(NPM/100 mL'ㅗ) & $3,4 \times 10^{5}$ & $7,4 \times 10^{4}$ & $3,8 \times 10^{3}$ & $\mathrm{Nd}$ \\
\hline \multirow{2}{*}{ Teste 4} & Coliformes totais (NPM.100 mL'ㄱ) & $1,9 \times 10^{6}$ & $5,4 \times 10^{4}$ & $8,0 \times 10^{5}$ & $\mathrm{Nd}$ \\
\hline & Escherichia coli (NPM/100 mL'1') & $1,2 \times 10^{6}$ & $5,5 \times 10^{6}$ & $4,0 \times 10^{4}$ & $\mathrm{Nd}$ \\
\hline \multirow{2}{*}{ Teste 5} & Coliformes totais (NPM.100 mL'1) & $3,5 \times 10^{6}$ & $4,5 \times 10^{2}$ & $7,4 \times 10^{5}$ & $\mathrm{Nd}$ \\
\hline & Escherichia coli (NPM/100 mL'1) & $23,3 \times 10^{4}$ & $9,5 \times 10^{3}$ & $3,6 \times 10^{3}$ & $\mathrm{Nd}$ \\
\hline Teste 7 & Escherichia coli (NPM/100 mL'1) & $1,0 \times 10^{5}$ & $1,2 \times 10^{2}$ & $8,8 \times 10^{4}$ & $\mathrm{Nd}$ \\
\hline \multirow{2}{*}{ Teste 8} & Coliformes totais (NPM.100 mL') & $4,5 \times 10^{5}$ & $6,6 \times 10^{4}$ & $9,5 \times 10^{5}$ & $\mathrm{Nd}$ \\
\hline & Escherichia coli (NPM/100 mL'1) & $1,6 \times 10^{5}$ & $6,5 \times 10^{4}$ & $8,8 \times 10^{4}$ & $\mathrm{Nd}$ \\
\hline \multirow{2}{*}{ Teste 9} & Coliformes totais (NPM.100 mL'ㄱ) & $5,1 \times 10^{5}$ & $5,9 \times 10^{3}$ & $4,3 \times 10^{4}$ & $\mathrm{Nd}$ \\
\hline & Escherichia coli (NPM/100 mL-1) & $2,6 \times 10^{5}$ & $6,1 \times 10^{4}$ & $8,3 \times 10^{2}$ & $\mathrm{Nd}$ \\
\hline \multirow{2}{*}{ Teste 10} & Coliformes totais (NPM.100 mL'1) & $6,1 \times 10^{5}$ & $2,7 \times 10^{4}$ & $7,9 \times 10^{3}$ & $\mathrm{Nd}$ \\
\hline & Escherichia coli (NPM/100 mL'1) & $3,3 \times 10^{5}$ & $4,7 \times 10^{4}$ & $1,7 \times 10^{5}$ & $\mathrm{Nd}$ \\
\hline
\end{tabular}


aplicados à massa líquida estão em escala compatível com as vibrações das células, são transmitidos apenas movimentos oscilatórios para elas sem causar ruptura. Entretanto, à medida que esse movimento oscilatório excede a força da membrana da célula causa sua ruptura, ampliando o efeito da desinfecção no meio líquido.

\section{CONCLUSÃO}

O controle da vazão da água de alimentação do reator de cavitação associado ao número de orifícios e à velocidade de homogeneização tem influência direta na estabilização da temperatura de ebulição da água. O reator de cavitação operando com $8.200 \mathrm{rpm}$, contendo 26 orifícios na face superior do rotor a uma vazão de 60 L.h ${ }^{-1}$, após 21 minutos de funcionamento, estabilizou-se, mantendo constante a temperatura de ebulição da água tratada, reduzindo significativamente concentrações de vários parâmetros físico-químicos bem como de indicadores de contaminação microbiológica, destacando-se as reduções de nitrito e nitrato e a inativação completa dos micro-organismos estudados (coliformes totais e Escherichia coli), alcançando níveis de potabilidade.

\section{REFERÊNCIAS}

AMERICAN PUBLIC HEALTH ASSOCIATION. (1998) Standard Methods for the Examination of Water and Waste Water. $20^{\text {th }}$ edition. Washington: American Public Health Association (APHA), the American Water Works Association (AWWA) and the Water Environment Federation (WEF), 1325 p.

ARROJO, S. \& BENITO, Y. (2008) A theoretical study of hydrodynamic cavitation. Ultrasonics Sonochemistry, v. 15, n. 3, p. 203-211.

BRASIL. (2005) Ministério da Saúde. Secretaria de Vigilância em Saúde. Coordenação-Geral de Vigilância em Saúde Ambiental. Portaria MS n. ${ }^{\circ}$ 518/2004. Brasília: Editora do Ministério da Saúde.

CAUPIN, F. \& HERBERT, E. (2006) Cavitation in water: a review. Comptes Rendus Physique, v. 7, p. 1000-1017.

DELMÉE, G.J. (2003) Manual de medição de vazão. 3 ed. São Paulo: Edgard Blucher Ltda., $346 \mathrm{p}$.

DOULAH, M.S. \& HAMMOND, T.H. (1975) A hydrodynamic mechanism for the disintegration of Saccharomyces cerevesiae in an industrial homogenizer. Biotechnology and Bioengineering, v. 17, p. 845-58.

FERREIRA FILHO, S.S. \& SAKAGUTI, S. (2008) Comportamento cinético do cloro livre em meio aquoso e formação de subprodutos da desinfecção. Revista de Engenharia Sanitária e Ambiental, v. 13, n. 2, p. 198-206.

GOGATE, P.R. (2007) Application of cavitational reactors for water disinfection: Current status and path forward. Journal of Environmental Management, v. 85, p. 801-815.

GOGATE, P.R.; SHIRGAONKAR, I.Z.; SIVAKUMAR M.; SENTHILKUMAR P.; VICHARE N.P.; PANDIT A.B. (2001) Cavitation Reactors: Efficiency Assessment Using a Model Reaction. AlChE Journal, v. 47, n. 11, p. 2526-2538.

GOGATE P.R.; TAYAL, R.K.; PANDIT, A.B. (2006) Cavitation: A technology on the horizon. Current Science, v. 91, n. 1, p. 35-46.
JYOTI, K.K. \& PANDIT, A.B. (2001) Water disinfection by acoustic and hydrodynamic cavitation. Biochemical Engineering Journal, v.7, p. 201-212.

JYOTI, K.K. \& PANDIT, A.B. (2003) Hybrid cavitation methods for water disinfection. Biochemical Engineering Journal, v. 14, p. 9-17.

JYOTI, K.K. \& PANDIT, A.B. (2004) Ozone and cavitation for water disinfection. Biochemical Engineering Journal, v. 18, p. 9-19.

MASON, T.J. (1992) Practical Sonochemistry: Users Guide in Chemistry and Chemical Engineering (Ellis Horwood Series in Organic Chemistry). Chichester, UK: Ellis Horwood, 150 p.

PASCHOALATO, C.F.P.R.; TRIMAILOVAS, M.R; BERNADO, L. (2008) Formação de subprodutos orgânicos halogenados nas operações de pré-oxidação com cloro, ozônio e peroxônio e pós-cloração em água contendo substância húmica. Revista de Engenharia Sanitária e Ambiental, v. 13, n. 3, p. 313-322.

RODIER, J. (1990) Análisis de las aguas: aguas naturales, aguas residuales, agua de mar. Barcelona: Omega, 1990, 1059 p.

SOUZA, J.B. \& DANIEL, L.A. (2005) Comparação entre hipoclorito de sódio e ácido peracético na inativação de $E$. coli, colifagos e $C$. perfringens em água com elevada concentração de matéria orgânica. Revista de Engenharia Sanitária e Ambiental, v. 10, n. 2, p. 111-117.

SUSLICK, K.S.; DIDENKO, Y.; FANG, M.M.; HYEON, T.; KOLBECK, K.J.; MCNAMARA III, W.B.; MDLELENI, M.M.; WONG, M. (1999) Acoustic cavitation and its chemical consequences. Philosophical Transactions of the Royal Society A: Physical, Mathematical and Engineering Sciences The Royal Society, v. 357, p. 335-353.

TEIXEIRA, C.P.A.B. \& JARDIM, W.F. (2004) Processos oxidativos avançados, conceitos teóricos. Caderno temático. Universidade Estadual de Campinas (Unicamp), v. 3, p. 1-83. 